\title{
Micropogon furnieri: FECUNDIDADE E TIPO DE DESOVA
}

\author{
(Recebido em 9/12/1968)
}

\author{
A. E. A. DE M. Vazzoler
}

Instituto Oceanográfico da Universidade de São Paulo

\section{SYNOPSIS}

Micropogon furnieri (Desmarest, 1822) Jordan, 1884 is one of the species of fish of commercial importance occurring along the southern coast of Brazil. It has been the subject of several studies and some aspects of its life cicle, behavior and abundance are already known. This is a contribution to the knowledge of some aspects of the reproduction of the species. The type of spawning is discussed and fecundity has been evaluated and shown to be dependent on body length, not on body weight.

\section{INTRODUÇÃO}

Micropogon furnieri (DESMAREST, 1822) JoRDAN, 1884 (corvina) é um peixe de grande importância econômica na costa centro-sul do Brasil, sendo capturado em quantidades consideráveis (RICHARDSON \& Moraes, 1960; Vazzoler \& SÁ, 1963). Alguns aspectos de seu ciclo de vida, comportzmento e abundância já foram estudados (VAzzoler, G., 1962; Franco, 1959; Vannucci, 1963; Vazzoler, 1963a; Vazzoler \& SAntos, 1965; VAzzoler, 1965). Porém, não há dados sôbre o tipo de desova e fecundidade dessa espécie na costa do Brasil. Tais informações são essenciais ao conhecimento da biologia da espécie e à correta avaliação quantitativa de seus estoques.

Esta é uma contribuição ao conhecimento do tipo de desova e da fecundidade de $M$. furnieri da costa sul do Brasil. É uma etapa dos estudos sôbre fecundidade de peixes da costa centro-sul do Brasil, projeto a longo prazo, que conta com apoio da Fundação de Amparo à Pesquisa do Estado de São Paulo.

\section{MATERIAL E MÉTODOS}

O material selecionado para êste estudo constou de 56 exemplares de $M$. furnieri, coletados durante o período de setembro de 1963 a março de 1964, na costa sul do Brasil, entre as latitudes $29^{\circ} \mathrm{S}$ e $33^{\circ} \mathrm{S}$.

Todos os exemplares (fêmeas) apresentavam-se no estádio "maduro", sendo que um encontrava-se em fase inicial e outro em fase finar dêsse estádio. Tra-

* Trabalho realizado com apoio financeiro da Fundação de Amparo à Pesquísa do Estado de São Paulo. Publ. n.o 271 do Inst. Ocean. da USP. balhamos sòmente com os 54 exemplares restantes que não se encontravam plenamente "maduros"; tal precaução foi tomada para que não houvesse a possibilidade de ter ocorrido desova parcial (eliminação de parte dos óvulos "maduros"), o que implicaria numa sub-estimativa da fecundidade.

Dêsses exemplares foram verificados o comprimento total (em centímetros), o pêso total e o pêso dos ovários (em gramas). O intervalo de comprimento abrangido foi de 32,5 a $59,5 \mathrm{~cm}$.

A coleta dos ovários e a dissociação dos mesmos seguiram as normas relatadas em trabalho anterior (VAzzoler, 1963b). Uma vez dissociadas as gônadas, para a eliminação dos resíduos dos tecidos, utilizamo-nos de uma série de peneiras granulométricas com malhas de $1410,1000,710,500,250$ e 125 micra, empilhadas e dispostas em ordem decrescente do tôpo para a base. As gônadas dissociadas foram colocadas na peneira superior (1410 micra) e lavadas com água corrente, usando-se um tubo de borracha com um pequeno chuveiro na extremidade. De modo geral os resíduos são retidos nas duas primeiras peneiras, se o material não tiver sido mantido por tempo excessivo na solução de GiLson. A quase totalidade dos ovocitos jovens (p. 28) foi eliminada, pois passam através das malhas da última peneira (125 micra), o que facilita o trabalho subseqüente.

As frações da massa de óvulos dissociados que permaneceram nas peneiras foram recolhidas, e êsse material foi manipulado segundo método estabelecido no trabalho anterior (VAzZOLER, op. cit.).

Os óvulos foram medidos com ocular micrométrica, com aumento de 120 vêzes; as medidas foram 
agrupadas em intervalos de 84,37 micra (valor de uma divisão da ocular micrométrica). As distribuições de freqüência dos diâmetros dos óvulos foram agrupadas segundo a posição da última moda (VAZZOLER, op. cit.) como está mostrado na Figura 1. 0 número entre parêntesis indica a freqüência de exemplares agrupados em cada fase.

Para estimar-se a fecundidade é necessário determinar que categorias de tamanhos de óvulos existentes nas gônadas são eliminadas durante o período de desova. Com base em observações de cortes histológicos de ovários dessa espécie (p. 28) foram considerados, na estimativa da fecundidade, todos os óvulos com diâmetro superior a 300 micra.

Foi calculado o fator de condição $K$ (VAzzoLER \& VAzzoler, 1965) para cada um dos exemplares.

Os dados sôbre os quais é baseado o presente trabalho estão apresentados na Tabela I.

Foram estudadas regressões entre os quatro primeiros parâmetros constantes da tabela acima citada e feita uma análise de regressão múltipla entre o logarítmo do comprimento, o logarítmo do pêso e a fecundidade. Para êsses cálculos foram considerados apenas os 54 exemplares em fase intermediária do estádio "maduro". Os resultados estão apresentados na Tabela II.

\section{RESULTADOS E DISCUSSÃO}

\section{a) Desova}

Estudos em andamento sôbre o processo de maturação sexual de $M$. furnieri (VAZzoler, em preparação) baseados principalmente na análise de cortes histológicos de gônadas nos vários estádios de desenvolvimento, mostraram que:

a) em gônadas imaturas ocorrem apenas ovocitos jovens, com diâmetro sempre inferior a $150 \mathrm{mi}$ cra, avitelogênicos, poliédricos, de citoplasma homogêneo;

b) em gônadas que estão iniciando o processo de maturação observam-se ovocitos em fases mais avançadas, com diâmetro mínimo de 170 micra, que já apresentam granulações vitelínicas no citoplasma;

c) em gônadas "maduras" (fases iniciais) ocor. rem, além dos ovocitos jovens e dos em fase mais avançada, óvulos com diâmetro mínimo de $300 \mathrm{mi}$ cra, com granulações vitelínicas bem desenvolvidas;

d) em gônadas totalmente esvaziadas subsistem óvulos residuais murchos e ovocitos avançados, com sinais de necrose, ao lado de ovocitos jovens que serão o ponto de partida para novas desovas.

Assim sendo, há indicações de que, durante um período de desova, todos os óvulos com diâmetro acima de 300 micra completam seu desenvolvimento e são eliminados.

A análise das distribuições de freqüência de diâmetros de óvulos das gônadas maduras (Fig. 1) mostra o desenvolvimento de grupos sucessivos de óvulos. A última curva (apenas 1 exemplar) refere-se ao exemplar em fase final do estádio "maduro".
A existência dêsses grupos, associada às observações histológicas, sugere que a desova de $M$. furnieri é parcelada, ocorrendo mais de uma vez durante o período de desova (Yuen, 1955; Howard \& LANDA, 1958).

TABELA I - Dados sôbre comprimento total em $\mathrm{cm}\left(\mathrm{L}_{\mathrm{t}}\right)$, pêso total $\left(\mathrm{W}_{\mathrm{t}}\right)$ e pêso das gônadas $\left(\mathrm{W}_{\mathrm{g}}\right)$ em $g$, fecundidade estimada (F) em milhares de óvulos e fator de condição $\left(\mathrm{K}=\frac{\mathrm{W}_{\mathrm{t}}}{\mathrm{L}_{\mathrm{t}}^{3}} \cdot 10^{3}\right)$, referentes a Micropogon furnieri da costa sul do Brasil

\begin{tabular}{|c|c|c|c|c|c|}
\hline N.o & $L_{t}$ & $\mathrm{w}_{\mathrm{t}}$ & $\mathrm{W}_{\mathrm{g}}$ & F & K \\
\hline CF 1 & 59,0 & $2.110,0$ & 76,0 & 776.006 & 10 \\
\hline CF 2 & 52,0 & $1.675,0$ & 31,0 & 278.596 & 11 \\
\hline CF 3 & 45,5 & $1.000,0$ & 47,0 & 587.648 & 10 \\
\hline CF 4 & 45,0 & 990,0 & 39,0 & 496.611 & 10 \\
\hline CF 5 & 47,0 & $1.250,0$ & 65,0 & 704.719 & 12 \\
\hline CF 6 & 44,0 & 935,0 & 16,0 & 186.987 & 10 \\
\hline CF 7 & 41,5 & 860,0 & 27,0 & 262.146 & 12 \\
\hline CF 9 & 51,0 & $1.740,0$ & 94,0 & 714.901 & 13 \\
\hline CF 10 & 46,0 & $1.185,0$ & 49,0 & 574.822 & 12 \\
\hline CF 11 & 43,0 & 865,0 & 14,0 & 237.226 & 10 \\
\hline CF 12 & 43,5 & 965,0 & 26,0 & 271.231 & 11 \\
\hline CF 13 & 46,0 & $1.060,0$ & 28,0 & 358.094 & 10 \\
\hline CF 14 & 44,0 & 835,0 & 18,0 & 344.680 & 9 \\
\hline CF 15 & 38,5 & 620,0 & 19,0 & 296.000 & 10 \\
\hline CF 16 & 46,0 & 995,0 & 26,0 & 643.368 & 10 \\
\hline CF 17 & 37,0 & 575,0 & 14,0 & 204.680 & 11 \\
\hline CF 18 & 37,0 & 575,0 & 8,0 & 240.934 & 11 \\
\hline CF 19 & 34,0 & 455,0 & 8,0 & 247.931 & 11 \\
\hline CF 20 & 32,5 & 365,0 & 4,0 & 44.733 & 10 \\
\hline CF 21 & 37,0 & 595,0 & 15,0 & 246.490 & 11 \\
\hline CF 23 & 40,0 & 675,0 & 6,0 & 211.054 & 10 \\
\hline CF 24 & 43,5 & $1.025,0$ & 14,0 & 139.771 & 12 \\
\hline CF 25 & 40,5 & 745,0 & 10,0 & 105.702 & 11 \\
\hline CF 26 & 41,0 & 765,0 & 5,0 & 158.776 & 11 \\
\hline CF 28 & 43,5 & 805,0 & 10,0 & 182.903 & 9 \\
\hline CF 29 & 43,0 & 840,0 & 19,0 & 209.524 & 10 \\
\hline CF 30 & 42,0 & 895,0 & 32,0 & 352.882 & 12 \\
\hline CF 31 & 43,0 & 830,0 & 20,0 & 582.412 & 10 \\
\hline CF 33 & 47,0 & $1.385,0$ & 45,0 & 237.320 & 13 \\
\hline CF 34 & 42,5 & 770,0 & 29,0 & 703.725 & 10 \\
\hline CF 35 & 51,0 & $1.490,0$ & 31,0 & 600.838 & 11 \\
\hline CF 36 & 47,5 & 1. 228,0 & 22,0 & 666.000 & 11 \\
\hline CF 37 & 53,5 & $2.100,0$ & 105,0 & 467.770 & 13 \\
\hline CF 38 & 55,5 & 1. 866,0 & 51,0 & 650.393 & 10 \\
\hline CF 39 & 55,5 & 1. 778,0 & 48,0 & 310.310 & 10 \\
\hline CF 44 & 49,0 & $1.230,0$ & 65,0 & 370.544 & 10 \\
\hline CF 45 & 53,0 & $1.530,0$ & 30,0 & 341.112 & 10 \\
\hline CF 46 & 54,0 & 1. 720,0 & 105,0 & 735.369 & 10 \\
\hline CF 48 & 56,5 & $1.886,0$ & 66,0 & 481.104 & 10 \\
\hline CF 51 & 39,5 & 680,0 & 32,0 & 375.434 & 11 \\
\hline CF 53 & 42,5 & 810,0 & 18,0 & 193.654 & 10 \\
\hline CF 54 & 49,0 & $1.245,0$ & 25,0 & 183.915 & 10 \\
\hline CF 55 & 45,0 & 965,0 & 21,0 & 639.540 & 10 \\
\hline CF 57 & 42,5 & 725,0 & 26,0 & 728.280 & 9 \\
\hline CF 58 & 43,5 & 860,0 & 9,5 & 529.010 & 10 \\
\hline CF 59 & 40,0 & 725,0 & 18,5 & 346.626 & 11 \\
\hline CF 61 & 41,5 & 790.0 & 20,0 & 246.391 & 11 \\
\hline CF 62 & 40,0 & 680,0 & 14,0 & 184.580 & 10 \\
\hline CF 63 & 45,0 & 920,0 & 22,0 & 267.199 & 10 \\
\hline CF 65 & 41,0 & 715,0 & 16,0 & 184.972 & 10 \\
\hline CF 66 & 48,0 & $1.530,0$ & 26,5 & 262.024 & 13 \\
\hline CF 67 & 47,0 & $1.130,0$ & 26,5 & 242.667 & 10 \\
\hline CF 68 & 44,0 & 939,0 & 29,0 & 256.821 & 11 \\
\hline CF 69 & 49,0 & $1.250,0$ & 47,0 & 415.538 & 10 \\
\hline CF 8 & 56,5 & $3.420,0$ & 54,0 & Inicial & 18 \\
\hline CF 56 & 47,5 & 925,0 & 25,0 & Final & 3 \\
\hline
\end{tabular}


TABELA II - Parâmetros das regressões obtidas entre fecundidade (F) e logaritmo do comprimento total (Lt), logarítmo do pêso total (Wt) e pêso das gônadas (Wg)

\begin{tabular}{l|c|c|c|c|c}
\hline \multicolumn{1}{c|}{ Regressão } & $\mathrm{N}$ & $\mathrm{Ax}$ & $\mathrm{b}$ & $\mathrm{a}$ & $\mathrm{r}^{2}$ \\
\hline \hline F/log Lt & 54 & $32,5-59,5$ & $190,3 \pm 43,0$ & $-276,4 \pm 71,0$ & 0,523 \\
F/log Wt & 54 & $365,0-2.110,0$ & $57,1 \pm 14,2$ & $-133,2 \pm 42,4$ & 0,488 \\
F/Wg & 54 & $4,0-105,0$ & $50,2 \pm 0,9$ & $2.187,7 \pm 36,0$ & 0,363 \\
\hline
\end{tabular}

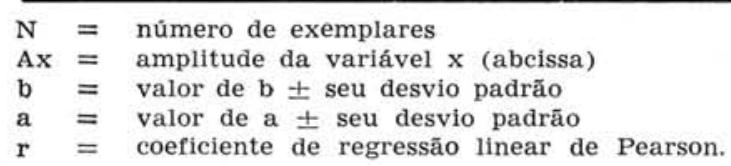

Esse tipo de desova foi observado para outras espécies de peixes do Atlântico Sul Ocidental, como Merluccius merluccius hubbsi (ANGELESCU et al., 1958; Ciechomsкi, 1967) e Macrodon ancylodon (Vazzoler, 1963b; Yamaguti, 1967).

\section{b) Fator de condição}

Está demonstrado através de vários estudos que o valor do fator de condição $K$ varia durante o ciclo sexual de peixes (Clark, 1934; Angelescu et al., op. cit.; Fuster de Plaza \& Boschi, 1961; VazzoLER \& VAZZOLER, 1965) e que reflete o estado fisiológico dos mesmos. Durante o processo de matura̧̧ão ocorre uma queda $\mathrm{em}$ seu valor, provàvelmente devido ao consumo de reservas graxas durante êsse período (BAGENAL, 1957).

Segundo Holliday (1960), em peixes adultos, principalmente nos estados iniciais de formação de ovocitos, o fator alimento é de grande importância. Em geral, boas condições alimentares refletem-se num aumento da fecundidade.

De acôrdo com Hempel (1965), um valor elevado de $K$, em alguns casos, pode resultar de alimentação não balanceada, não favorecendo o desenvolvimento de maior número de óvulos. Assim sendo, nem sempre um alto valor de $K$ está relacionado a fecundidade mais elevada.

MacGregor (1957) mostrou que para Sardinops caerulea do Pacífico, quando ocorrem dois exemplares do mesmo comprimento, o de maior pêso (portanto com $K$ mais elevado) contém mais óvulos.

Ciechomski (1967), estudando o tipo de desova e a fecundidade da merluza da costa da Argentina, verificou não haver correlação entre fator de condição e fecundidade.

Pela análise dos dados da Tabela I verifica-se que dentro do estádio "maduro" ocorrem diferentes valôres para $K$, segundo a fase em que se encontra o exemplar, dentro dêsse estádio. $O$ valor de $K$ é elevado para o exemplar em fase inicial do estádio (CF 8), e baixo para o em fase final (CF 56); para os exemplares em fases intermediárias, os valôres distribuem-se numa faixa relativamente homogênea, não havendo variação com o comprimento total, encontrando-se, portanto, êsses exemplares em estados fisiológicos similares. Verifica-se ainda que para $\mathrm{Mi}$. cropogon furnieri não existe correlação entre os valôres individuais de fecundidade e fator de condição.

\section{c) Fecundidade}

As relações entre: a) fecundidade/comprimento total (dez milhares de óvulos/cm), b) fecundidade/ pêso total (dez milhares de óvulos/g), e c) fecundidade/pêso das gônadas (dez milhares de óvulos/g), para os 54 exemplares estudados, estão representadas gràficamente nas Figuras 2, 3 e 4, respectivamente.

Há muita discussão sôbre qual o tipo de linha que melhor expressa essas relações (Simpson, 1951; Beverton \& Holt, 1957; MacGregor, 1957; BageNal, 1957; Peterson, 1961; Joseph, 1963; Wydoski \& COOPER, 1966; Topp, 1968).

Segundo MacGregor (op. cit.), quando a amplitude de comprimentos utilizados no cálculo da regressão da fecundidade sôbre o comprimento total não é muito grande, a curvilinearidade que poderia ocorrer não se torna evidente. É possível que no presente caso isso tenha ocorrido, pois a linha que melhor se ajustou aos dados foi uma reta, expressa por uma equação $\log$-arit, do tipo $\mathrm{Y}=\mathrm{a}+\mathrm{b} \log \mathrm{X}$.

A relação entre fecundidade e pêso total é também melhor descrita por uma reta expressa por equação do tipo acima citado.

A relação entre fecundidade e pêso das gônadas é expressa por uma reta $(\mathrm{Y}=\mathrm{a}+\mathrm{bX})$ e foi estabelecida com o intuito de poder ser usada para previsão da fecundidade. Entretanto a correlação entre êsses parâmetros é muito baixa (Tab. II), sendo que apenas $36 \%$ da fecundidade é explicada pelo pêso das gônadas.

Para verificar qual o fator preponderante na determinação da fecundidade foi feita uma análise de regressão múltipla entre o lagarítmo do comprimento, o logarítmo do pêso e a fecundidade (tomada em unidades de 10.000 óvulos). A análise indicou que apenas o comprimento do peixe é relevante ao número de óvulos.

\section{CONCLUSÕES}

1 - Micropogon furnieri apresenta desova parcelada, indicada pela distribuição polimodal da freqüência de diâmetro dos óvulos; os óvulos são eliminados em lotes, durante um período de desova; 


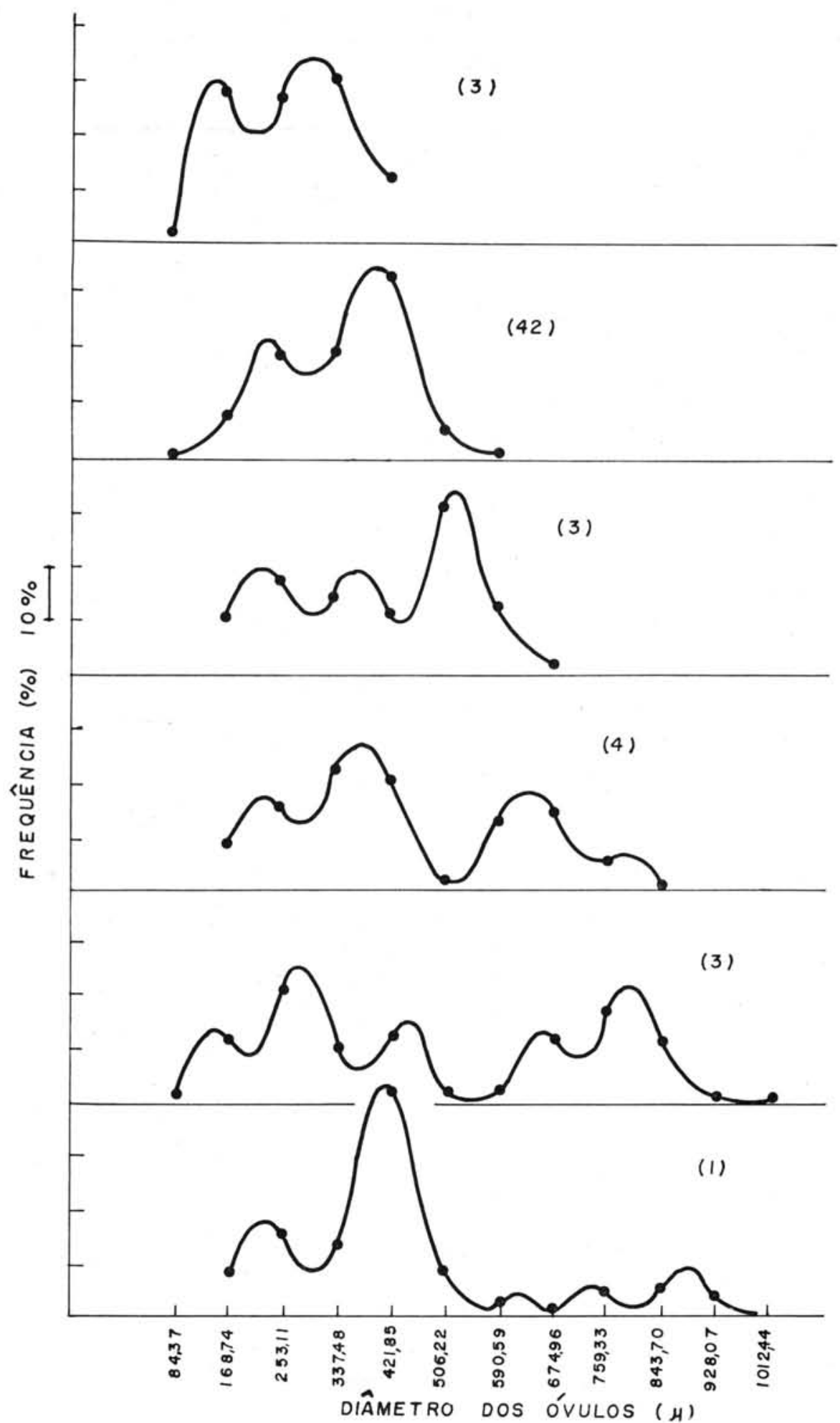

Fig. 1 - Distribuição de frequêencia dos diâmetros de óvulos de gônadas maduras, de Micropogon furnieri.

2 - As relações entre fecundidade e comprimento total e pêso total são melhores descritas por retas expressas por equações do tipo log-arit, sendo que a fecundidade depende do comprimento do peixe, e não de seu pêso;

3 - Para $M$. furnieri não se verificou a existência de correlação entre valôres individuais do fator de condição e fecundidade;

4 - A correlação entre fecundidade e pêso das gô. nadas é baixa $(36 \%)$ não sendo possível utilizar a regressão entre êsses dois parâmetros para previsão da fecundidade. 


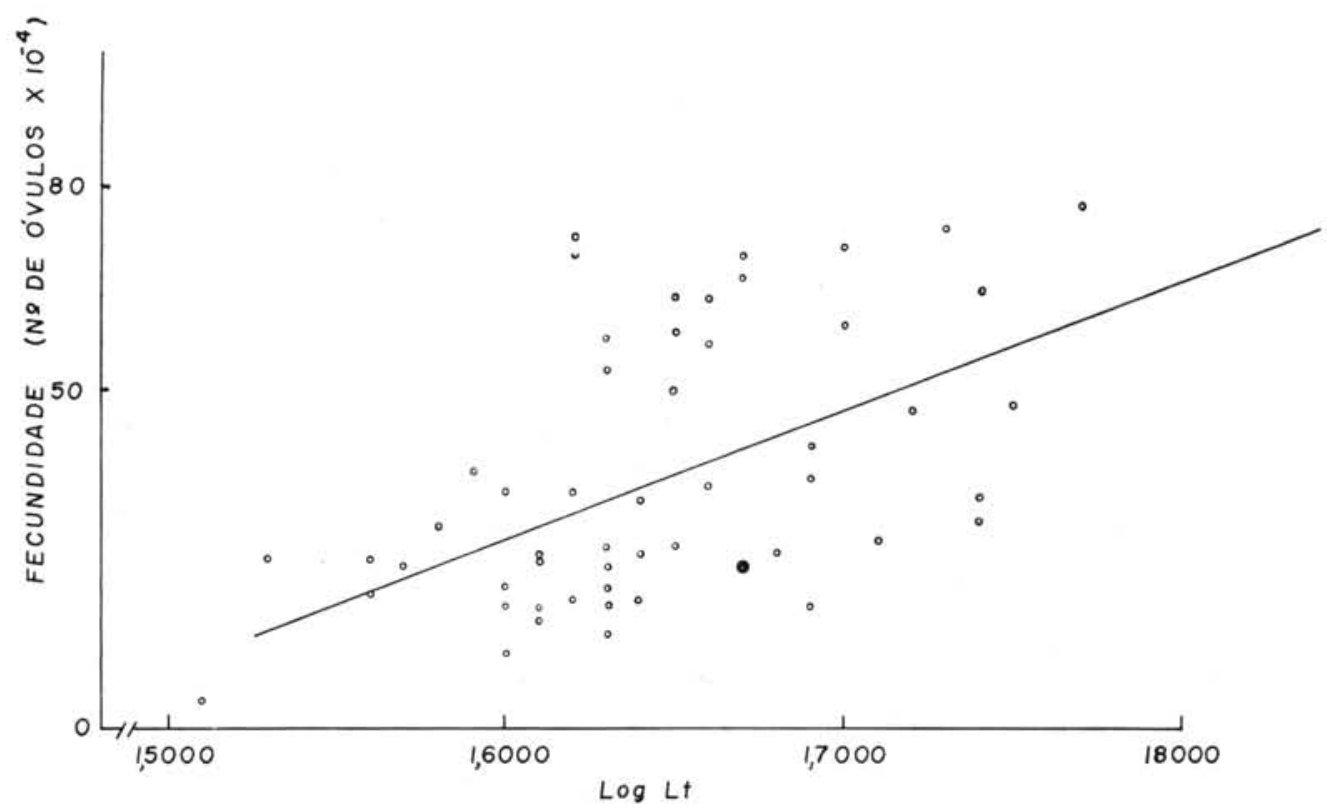

Fig. 2 - Representação gráfica da relação entre fecundidade e $\log$ do comprimento total.

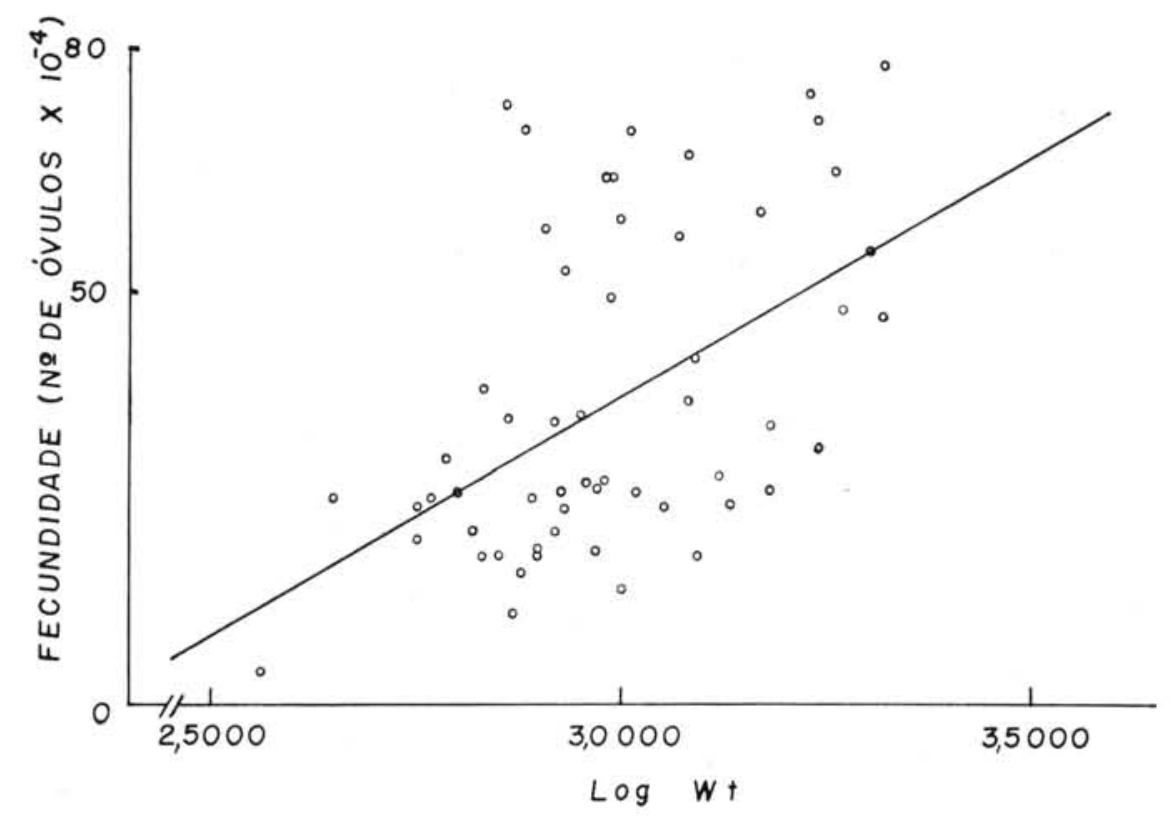

Fig. 3 - Representação gráfica da relação entre fecundidade e $\log$ do pêso total.

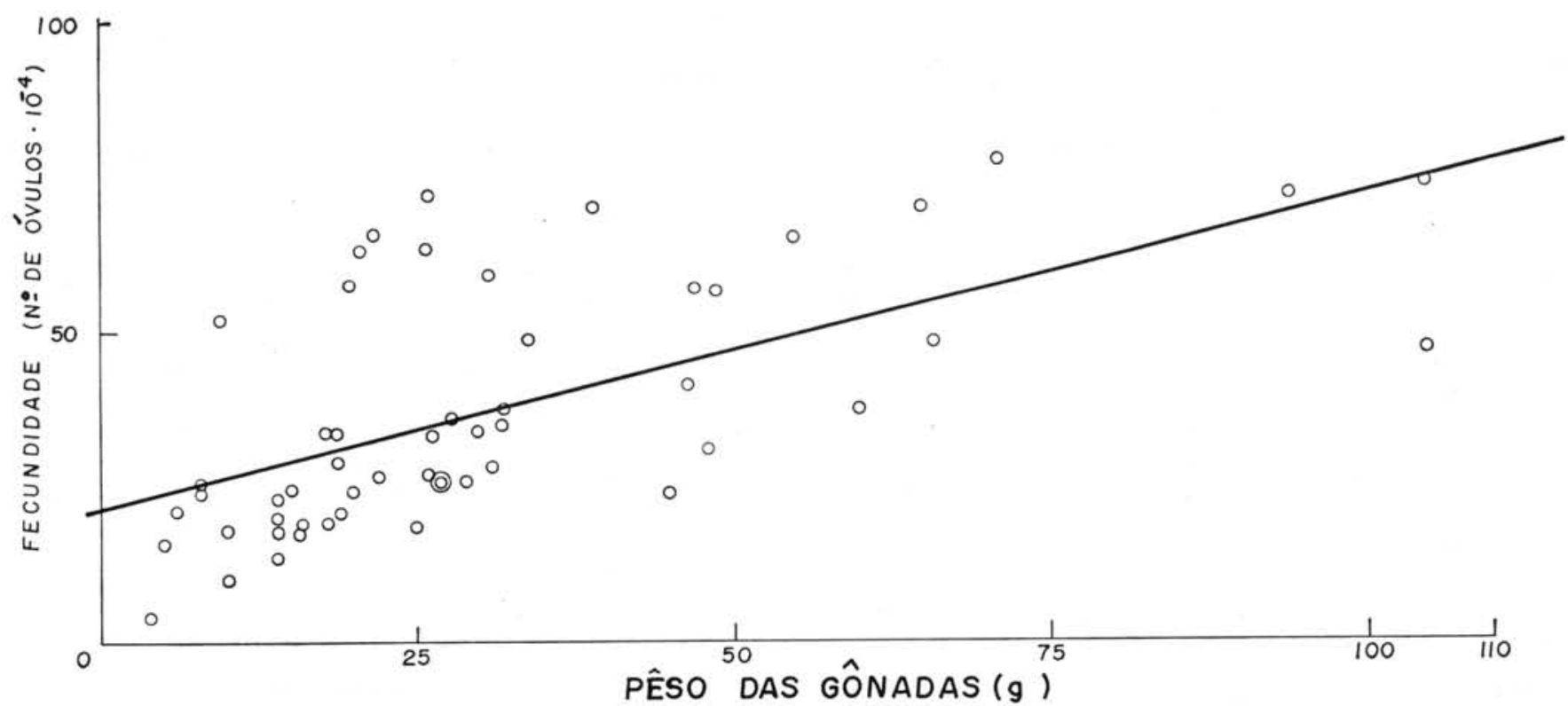

Fig. 4 - Representação gráfica da relação entre fecundidade e pêso das gônadas. 


\section{AGRADECIMENTOS}

O autor agradece ao Prof. Dr. P. E. Vanzolini pela orientação prestada na análise estatística dos resultados.

\section{B I B L I O G R A F I A}

ANgelescu, V., GNeri, F. S. \& NANi, A.

1958. La merluza del mar argentino. Argentina, Secret. Mar., Serv. Hidrogr. Nav., H 1004, 224 p.

BAgENAL, T. B.

1957. The breeding and fecundity of the long rough dab Hippoglossoides platessoides (Fabr.) and the associated cycle in condition. J. mar. biol. Ass. U.K., vol. 36, n. $^{\circ} 2$, p. 339 .

Beverton, R. J. H. \& Holt, S. J.

1957. On the dynamics of exploited fish populations. Fishery Invest. Lond., ser. 2, vol. 19, 535 p.

Ciechomski, J. D. de

1967. Caracter del desove y fecundidad de la merluza argentina, Merluccius merluccius hubbsi, del sector bonaerense. Boln Inst. Biol. mar., n. ${ }^{0} 13,30 \mathrm{p}$.

Clark, F. N.

1934. Maturity of the California sardine (Sardina caerulea), determined by ova diameter measurements. Fish Bull. Calif., n. ${ }^{\circ} 42,49 \mathrm{p}$.

Franco, G. T.

1959. Nota preliminar sôbre alimentação de alguns peixes comerciais brasileiros. Anais Acad. bras. Cienc., vol. $31, \mathrm{n}^{\circ} 4$, p. 589-593.

Fuster de Plaza, M. L. \& Boschi, E. E.

1961. Áreas de migración y ecología de la anchoa Lycengraulis olidus (Günther) en las aguas argentinas. Contrnes cient. Fac. Cienc, exact, fis, nat. Univ. B. Aires, ser. Zool., vol. 1, n. ${ }^{\circ}$ 3, $182 \mathrm{p}$.

Hempel, G.

1965. Fecundity and egg size in relation to the environment. Spec. Publs int. Commn NW Atlant. Fish., n. ${ }^{\circ} 6$.

HOLliday, F. G. T.

1960. The control of maturation in the herring. ICES, C.M., doc. n. 39 .

Howard, G. V. \& LANDA, A.

1958. A study of the age, growth, sexual maturity, and spawning of the anchoveta (Cetengraulis mysticetus) in the Gulf of Panama. Bull. inter-Am. trop. Tuna Commn, vol. 2, n. ${ }^{\circ}$, p. 389-437.

JoSEPH, J .

1963. Fecundity of yellowfin tuna (Thunnus albacares) and skipjack (Katsuwonus pelamis) from the eastern Pacific Ocean. Bull. inter-Am. trop. Tuna Commn, vol. 7, n. $^{\circ} 4$, p. $257-292$.

MacGregor, J. S .

1957. Fecundity of the Pacific sardine (Sardinops caerulea). Fishery Bull. Fish. Wildl. Serv. U.S., vol. 57, n. ${ }^{\circ} 121$, p. 427-449.
Peterson, C. L.

1961. Fecundity of the anchoveta (Cetengraulis mysticetus) in the Gulf of Panama. Bull, inter-Am. trop. Tuna Commn, vol. 6 , n. ${ }^{\circ}$, p. $55-68$.

Richardson, I. D. \& MoraEs, M. N. de

1960. A first appraisal of the landing and mechanism of the Santos fishery. Bolm Inst. oceanogr., vol. $11, \mathrm{n}^{\circ} 1$, p. 5-97.

Simpson, A. C.

1951. The fecundity of the plaice. Fishery Invest., Lond., ser. 2, vol. 17, n. ${ }^{\circ}$, 27 p.

Topp, R. W.

1968. An estimate of fecundity of the winter flounder, Pseudopleuronectes americanus. J. Fish. Res. Bd Can., vol, 25, n. ${ }^{\circ}$ 6, p. $1299-1302$.

VANNUCCI, M.

1963. Plâncton e ciclos alimentares. Contrções Inst, oceanogr. Univ. S Paulo, ser. Ocean. fis., n. ${ }^{\circ}$, p. $55-70$.

VAZZOLER, G.

1962. Sôbre a biologia da corvina da costa sul do Brasil. Bolm Inst. oceanogr., vol. 12, n. $^{\circ} 1$, p. $53-102$.

VAzzoler, A. E. A. de M.

1963a. Deslocamentos sazonais da corvina relacionados com as massas de água. Contrções Inst. oceanogr. Univ. S Paulo, ser. Ocean. biol., n. ${ }^{\circ} 5,8$ p.

1963b. Sôbre a fecundidade e desova da pescada-foguete. Bolm Inst. oceanogr., vol. 13 , n. 2 , p. $33-40$

1965. Estimativa da abundância relativa de corvina na costa centro-sul do Brasil. Bolm Inst. oceanogr., vol. $14, \mathrm{n}^{\circ}{ }^{\text {unni- }}$ co, p. 3-11.

Vazzoler, A. E. A. de M. \& SÁ, E. M. de

1963. Análise da pesca da corvina na costa centro-sul do Brasil. Bolm Inst. oceanogr., vol. 13, n. ${ }^{\circ} 2$, p. 61-70.

Vazzoler, A. E. A. de M. \& Santos, E. P.

1965. Migração da corvina, Micropogon furnieri, na costa sul do Brasil. Bolm Inst. oceanogr., vol. 14, n. $^{\circ}$ único, p. 125-128.

VAzzoler, A. E. A. de M. \& VAzzoler, G.

1965. Relation between condition factor and sexual development in Sardinella aurita (Cuv. \& Val., 1847). Anais Acad. bras. Cienc., vol. 37, suplem., p. 353-359.

Wydoski, R. S. \& CoOper, E. L.

1966. Maturation and fecundity of brook trout from infertile streams. J. Fish. Res. Bd. Can., vol. 23, n. $^{\circ}$ 5, p. $623-649$

Yamaguti, N.

1967. Desova da pescada-foguete, Macrodon ancylodon. Bolm Inst. oceanogr., vol. 16, n. ${ }^{\circ}$ único, p. 101-106.

YUEN, H. S. H.

1955. Maturity and fecundity of bigeye tuna in the Pacific. Spec. scient. Rep. U.S. Fish Wildl. Serv., Fisheries, n. ${ }^{\circ}$ 150, $30 \mathrm{p}$. 